Themed Issue: Drug-Induced Hypersensitivity Reactions

Guest Editor - Craig Svensson

\title{
Mechanisms of Drug-Induced Liver Injury
}

Submitted: August 26, 2005; Accepted: December 16, 2005; Published: February 3, 2006

Michael P. Holt ${ }^{1}$ and Cynthia Ju${ }^{1}$

${ }^{1}$ Department of Pharmaceutical Sciences, School of Pharmacy, University of Colorado Health Sciences Center, 4200 E 9th Ave, Box C-238, Denver, CO 80262

\section{Abstract}

The idiosyncratic nature and poor prognosis of drug-induced liver injury (DILI) make this type of reaction a major safety issue during drug development, as well as the most common cause for the withdrawal of drugs from the pharmaceutical market. The key to predicting and preventing DILI is understanding the underlying mechanisms. DILI is initiated by direct hepatotoxic effects of a drug, or a reactive metabolite of a drug. Parenchymal cell injury induces activation of innate and/or adaptive immune cells, which, in turn, produce proinflammatory and tissue hepatotoxic mediators, and/or mount immune reactions against drug-associated antigens. Understanding the molecular and cellular elements associated with these pathways can help identify risk factors and may ultimately facilitate the development of strategies to predict and prevent DILI.

KEYWORDS: Adverse drug reaction, hepatotoxicity, inflammation, innate immunity, adaptive immunity

\section{INTRODUCTION}

Drug-induced liver injury (DILI) is a major health problem that challenges not only health care professionals but also the pharmaceutical industry and drug regulatory agencies. According to the United States Acute Liver Failure Study Group, ${ }^{1}$ DILI accounts for more than $50 \%$ of acute liver failure, including hepatotoxicity caused by overdose of acetaminophen (APAP, 39\%) and idiosyncratic liver injury triggered by other drugs (13\%). Because of the significant patient morbidity and mortality associated with DILI, ${ }^{2}$ the U.S. Food and Drug Administration (FDA) has removed several drugs from the market, including bromfenac, ${ }^{3}$ ebrotidine, ${ }^{4}$ and troglitazone. ${ }^{5}$ Other hepatotoxic drugs, such as risperidone, trovafloxacin, and nefazodone, ${ }^{6,7}$ have been assigned "black box" warnings. DILI is the most common cause for the withdrawal of drugs from the pharmaceutical market. ${ }^{8}$

Corresponding Author: Cynthia Ju, Department of

Pharmaceutical Sciences, School of Pharmacy, University of Colorado Health Sciences Center, 4200 E 9th Ave, Box C-238, Denver, CO 80262. Tel: (303) 315-2180; Fax: (303) 315-6281; E-mail: Cynthia.Ju@UCHSC.edu
Although the exact mechanism of DILI remains largely unknown, it appears to involve 2 pathways - direct hepatotoxicity and adverse immune reactions. In most instances, DILI is initiated by the bioactivation of drugs to chemically reactive metabolites, which have the ability to interact with cellular macromolecules such as proteins, lipids, and nucleic acids, leading to protein dysfunction, lipid peroxidation, DNA damage, and oxidative stress. Additionally, these reactive metabolites may induce disruption of ionic gradients and intracellular calcium stores, resulting in mitochondrial dysfunction and loss of energy production. This impairment of cellular function can culminate in cell death and possible liver failure.

Hepatic cellular dysfunction and death also have the ability to initiate immunological reactions, including both innate and adaptive immune responses. Hepatocyte stress and/or damage could result in the release of signals that stimulate activation of other cells, particularly those of the innate immune system, including Kupffer cells (KC), natural killer (NK) cells, and NKT cells. These cells contribute to the progression of liver injury by producing proinflammatory mediators and secreting chemokines to further recruit inflammatory cells to the liver. It has been demonstrated that various inflammatory cytokines, such as tumor necrosis factor (TNF)- $\alpha$, interferon (IFN)- $\gamma$, and interleukin (IL)-1 $\beta,{ }^{9-11}$ produced during DILI are involved in promoting tissue damage. However, innate immune cells are also the main source of IL-10, IL-6, and certain postglandins, all of which have been shown to play a hepatoprotective role. ${ }^{12-14}$ Thus, it is the delicate balance of inflammatory and hepatoprotective mediators produced after activation of the innate immune system that determines an individual's susceptibility and adaptation to DILI.

In addition to the innate immune responses, clinical features of certain DILI cases strongly suggest that the adaptive immune system is activated and involved in the pathogenesis of liver injury. With regard to the involvement of the adaptive immune system in DILI, our current understanding is based on the hapten hypothesis and the $\mathrm{p}-\mathrm{i}$ ( $p$ harmacological interaction of drugs with immune receptors) concept. Evidence to support these hypotheses is gained by the detection of drug-specific antibodies and T cells in some patients with DILI. ${ }^{15-21}$

This review will focus on the involvement of the innate and adaptive immune systems in the pathogenesis of DILI. 


\section{DILI}

DILI can affect both parenchymal and nonparenchymal cells of the liver, leading to a wide variety of pathological conditions, including acute and chronic hepatocellular hepatitis, fibrosis/cirrhosis, cholestasis, steatosis, as well as sinusoidal and hepatic artery/vein damage. ${ }^{22}$ The predominant forms of DILI include acute hepatitis, cholestasis, and a mixed pattern. ${ }^{23}$ Acute hepatitis is defined as a marked increase in aminotransferases coinciding with hepatocellular necrosis. Cholestasis is characterized by jaundice with a concurrent elevation in alkaline phosphatase, conjugated bilirubin, and $\gamma$-glutamyl transpeptidase. Mixed-pattern DILI includes clinical manifestations of both hepatocellular and cholestatic injury.

The clinical diagnosis and prediction of DILI remain a major challenge due to various confounding factors. These factors include preexisting liver disease, multiple drug usage by patients, and most important, lack of reliable screening methods and diagnostic standards. As a general rule, alanine transaminase (ALT) levels greater than 3 times the upper limits of normal (ULN) have been identified as a marker for liver injury. Hyman Zimmerman noted that elevated ALT accompanied by jaundice was associated with a mortality between $5 \%$ and $50 \%{ }^{24}$ This observation has since been referred to as "Hy's rule" and is currently employed by the FDA in the evaluation of hepatotoxicity for newly developed drugs. However, it must be noted that $3 \times$ ULN of ALT levels are not necessarily predictive of overt severe liver toxicity or acute liver failure.

The occurrence of DILI ranges from 1 in 10000 to 1 in 100 $000 .^{22,25}$ Partly because of this low incidence, many hepatotoxic drugs fail to be detected during clinical trials, as the number of patients involved in a clinical study rarely exceeds between 2000 and 5000. It has been postulated that in order for a clinical study to detect a single case of DILI (with 95\% confidence), the study would require $\sim 30000$ patients. ${ }^{26} \mathrm{As}$ a result, better understanding of the underlying mechanisms of DILI is imperative for the development of improved preclinical screens and the design of more effective clinical trials to evaluate a drug's potential hepatotoxic effects before its release into the pharmaceutical market.

\section{MECHANISMS OF DILI}

\section{Drug-Induced Direct Hepatotoxicity}

Direct hepatotoxicity is often caused by the direct action of a drug, or more often a reactive metabolite of a drug, against hepatocytes. One classically studied drug used to examine the mechanisms of hepatotoxicity is APAP. APAP is a popular over-the-counter analgesic that is safe at therapeutic doses but at overdose can produce centrilobular hepatic necrosis, which may lead to acute liver failure. APAP is metabolized to a minor electrophilic metabolite, N-acetylp-benzoquinoneimine (NAPQI), ${ }^{27}$ which during APAP overdose depletes glutathione and initiates covalent binding to cellular proteins. These events lead to the disruption of calcium homeostasis, mitochondrial dysfunction, and oxidative stress ${ }^{28,29}$ and may eventually culminate in cellular damage and death. Fortunately, drug candidates that induce significant direct hepatotoxicity at therapeutic doses are more likely to be detected during preclinical toxicity screening and thus rarely reach the pharmaceutical market.

In most instances of DILI, it appears that hepatocyte damage triggers the activation of other cells, which can initiate an inflammatory reaction and/or an adaptive immune response. These secondary events may overwhelm the capacity of the liver for adaptive repair and regeneration, thereby contributing to the pathogenesis of liver injury.

\section{Drug-Induced Immune-Mediated Liver Injury}

Innate and Adaptive Immune System of the Liver

The innate immune system provides a first line of defense against microbial infection, but it is not sufficient in eliminating infectious organisms. The lymphocytes ( $T$ and $B$ cells) of the adaptive immune system provide a more versatile means of defense and possess "memory," which is the ability to respond more vigorously to repeated exposure to the same microbe. Moreover, cells of the innate immune system play an integral role in the initiation of adaptive immunity by presenting antigens and are crucial in determining the subsequent T-cell- or antibody-mediated immune response. Because of the liver's continuous exposure to pathogens, toxins, tumor cells, and harmless dietary antigens, it possesses a range of local immune mechanisms to cope with these challenges. The liver contains large numbers of both innate and adaptive immune cells, including the largest populations of tissue macrophages (KC), NK cells, and NKT cells. ${ }^{30}$ The liver also possesses a unique combination of intrahepatic lymphocytes, which include not only the conventional $\mathrm{CD}^{+}$and $\mathrm{CD} 8^{+} \mathrm{T}$ cells but also high percentages of $\gamma \delta \mathrm{T}$ cells and $\mathrm{CD} 4^{-} \mathrm{CD} 8^{-} \mathrm{T}$ cells. Collectively, the innate and adaptive immune cells contribute to the unique immune responses of the liver, including removal of pathogenic microorganisms, clearance of particles and soluble molecules from circulation, deletion of activated $\mathrm{T}$ cells, and induction of tolerance to food antigens derived from the gastrointestinal tract.

$\mathrm{KC}$ play an essential role in the phagocytosis and removal of pathogens entering the liver via portal-venous blood. Upon activation, $\mathrm{KC}$ produce various cytokines and other mediators, including prostanoids, nitric oxide, and reactive oxygen intermediates. These $\mathrm{KC}$ products play prominent roles in promoting and regulating hepatic inflammation, as well as modulating the phenotype of other cells in the liver, 


\section{The AAPS Journal 2006; 8 (1) Article 6 (http://www.aapsj.org).}

such as NK and NKT cells. ${ }^{31,32}$ Furthermore, KC represent a major population of antigen-presenting cells (APCs) within the liver. It has been demonstrated that $\mathrm{KC}$ can activate $\mathrm{T}$ cells in vitro, but they do so less efficiently than peritoneal-exudate macrophages. ${ }^{33}$ Studies of organ transplantation using animal models have further shown that inhibition of $\mathrm{KC}$ abrogated the prolonged survival of allografts induced by portal vein infusion of allogeneic donor cells. ${ }^{34-36}$ Collectively, this evidence suggests that $\mathrm{KC}$ play an important role in the delicate balance between the induction of immunity and tolerance within the liver.

Unique to the liver are the remarkably high frequencies of NK and NKT cells, which account for $\sim 50 \%$ of intrahepatic leukocytes. ${ }^{37}$ These cells act as a first line of defense against certain pathogens and invading tumor cells prior to the adaptive immune response of B and T lymphocytes. One characterized function of hepatic NK and NKT cells is their cytotoxic capacity against other cells. It has been demonstrated that freshly isolated liver NK cells spontaneously induce the cytotoxicity of various cell lines, whereas NKT cells are cytotoxic in the presence of IL-2. ${ }^{38}$ This cytotoxicity is further enhanced by IL-12 and IL-18, which are produced by activated KC. 31,32 Another function ascribed to NK and NKT cells is their ability to produce high levels of T helper (Th) 1 and Th2 cytokines upon stimulation. ${ }^{39} \mathrm{NK}$ cells have been shown to represent a major source of IFN- $\gamma$ in many types of liver disease ${ }^{30}$ NKT cells produce either IFN- $\gamma$ or IL-4, or in some cases both cytokines, depending on the differentiation state of the cells and the stimuli. ${ }^{38,40}$ It has also been demonstrated that IL-4 produced by NKT cells may be associated with the initiation and regulation of $\mathrm{Th} 2$ responses. ${ }^{41}$

The liver's adaptive immune responses are unique in that the liver is known to favor induction of immunological tolerance rather than immunity. This is supported by numerous studies demonstrating that (1) dietary antigens derived from the gastrointestinal tract are tolerized in the liver; (2) allogeneic liver organ transplants are accepted across major histocompatibility complex (MHC) barriers ${ }^{42}$; (3) preexposure to donor cells through the portal vein of recipient animals increased their acceptance of solid tissue allografts ${ }^{43,44}$; and (4) preexposure of soluble antigens via the portal vein leads to systemic immune tolerance. ${ }^{45,46}$ Several mechanisms have been suggested to account for this tolerance, including apoptosis of activated $\mathrm{T}$ cells, immune deviation, and active suppression.

The liver has been dubbed the "elephant's graveyard" for activated T cells. ${ }^{47}$ These cells accumulate in the liver before undergoing apoptosis. Studies using T-cell receptor transgenic models have demonstrated that following antigen exposure, the activated $\mathrm{T}$ cells undergo apoptosis after a transient accumulation within the liver. ${ }^{48,49}$ Immune deviation may account for liver-induced tolerance, as it has been shown that Th2 cytokine production is preferentially maintained when adoptively transferred Th1 and Th2 cells are recovered from the liver. ${ }^{50}$ It has also been reported that liver sinusoidal endothelial cells (LSEC) are capable of selectively suppressing IFN- $\gamma$-producing Th1 cells while concurrently promoting the outgrowth of IL-4-expressing Th2 cells. ${ }^{50}$ Active suppression of T-cell activation resulting in liver-induced tolerance is also likely to occur within the liver because of its unique anatomy and composition of "tolerogenic" APCs. Within the liver, blood flow slows down through the narrow sinusoids $(7-12 \mu \mathrm{m})$ and is temporarily obstructed by $\mathrm{KC}$, which reside in the sinusoidal lumen. Because of this reduction in blood flow, circulating $\mathrm{T}$ cells can interact with LSEC and KC. Consequently, naïve T cells, which would normally encounter APCs in lymphoid tissues, could be primed directly by LSEC and/or KC within the liver. Current evidence suggests that LSEC and $\mathrm{KC}$ as well as hepatic dendritic cells are important in the induction of tolerance, rather than the activation of T-cell responses. It has been further demonstrated that although LSEC are capable of presenting antigen to T cells, LSEC-activated $\mathrm{CD}^{+}$or $\mathrm{CD} 8^{+} \mathrm{T}$ cells fail to differentiate into Th1 cells or cytotoxic effector cells, respectively. ${ }^{51,52}$ In addition, studies have shown that $\mathrm{KC}$ and hepatic dendritic cells are not effective APCs when compared with their counterparts in lymphoid tissues. ${ }^{33,53}$

In summary, it is the unique composition of innate and adaptive immune cells in the liver and the characteristic response of the liver to endogenous and exogenous antigens that may account for the mechanism of immune-mediated DILI, as well as the low occurrence and unpredictable nature of these reactions.

\section{Role of Innate Immunity in DILI}

Drug-induced stress and/or damage of hepatocytes may trigger activation and inflammatory responses of the innate immune system within the liver. Evidence to support this idea has been mainly obtained from studies of liver injury induced by overdose of APAP, which is one of the few drugs that provide an experimental animal model of DILI. There is growing evidence that the initial NAPQI-induced hepatocyte damage may lead to activation of innate immune cells within the liver, thereby stimulating hepatic infiltration of inflammatory cells. Activated cells of the innate immune system produce a range of inflammatory mediators, including cytokines, chemokines, and reactive oxygen and nitrogen species that contribute to the progression of liver injury. Some of these mediators, such as IFN- $\gamma$, Fas, or Fas ligand, are directly involved in causing liver damage; mutant mice lacking these factors are resistant to APAP hepatotoxicity. ${ }^{11,54}$ On the other hand, the innate immune cells also represent a major source of hepatoprotective factors, as it has been demonstrated that transgenic mice deficient in IL-10, IL-6, or COX-2 are more susceptible to APAP-induced liver injury. ${ }^{12-14}$ 
The innate immune cells reported to participate in APAP hepatotoxicity include NK and NKT cells, macrophages, and neutrophils. A recent study demonstrated that depletion of NK and NKT cells protected mice from APAP-induced liver injury. ${ }^{54}$ This protective mechanism seems to involve eliminating the production of IFN- $\gamma$ and various other proinflammatory chemokines as well as decreasing neutrophil accumulation within the liver. APAP hepatotoxicity has also been attributed in part to the activation of $\mathrm{KC}$ secondary to hepatocyte damage ${ }^{55,56} \mathrm{KC}$ activation results in the release of a wide range of proinflammatory mediators, such as TNF- $\alpha$, which may directly induce tissue damage, and IL12 and IL-18, which are important activators of NK and NKT cells. However, other studies suggest that KC may play a protective role in addition to their protoxicant effect, as $\mathrm{KC}$ are the predominant source of IL-10 and IL-6, which are important in counteracting inflammatory responses and/or stimulating liver regeneration. ${ }^{57}$ Although many studies have shown neutrophil accumulation in the liver of APAP-treated animals, ${ }^{11,54,58,59}$ the pathogenic role of these cells remains controversial. One study has shown that in vivo injection of rabbit antineutrophil antiserum protected rats against APAP toxicity, ${ }^{59}$ while another study suggested that neutrophils are recruited into the liver to remove cellular debris and do not directly cause tissue damage. ${ }^{58}$

Our understanding of APAP-induced liver injury underscores the role of the innate immune system as an important regulator in the progression and severity of tissue damage. However, the temporal activation of different cell populations and their production of various mediators, as well as the interplay among these cells, warrant further investigation. A proposed mechanism of DILI involving parenchymal cell damage and subsequent activation of the innate immune system is presented in Figure 1.

\section{Role of Adaptive Immune Response in DILI}

The clinical features of some cases of DILI strongly suggest an involvement of the adaptive immune system. These clinical characteristics include (1) concurrence of rash, fever, and eosinophilia; (2) delay of the initial reaction (1-8 weeks) or requirement of repeated exposure to the culprit drug; (3) rapid recurrence of toxicity on reexposure to the drug; and (4) presence of antibodies specific for native or drug-modified hepatic proteins. ${ }^{23}$ Drugs suspected to induce these types of reactions include halothane, tienilic acid, dihydralazine, diclofenac, phenytoin, and carbamazepine. ${ }^{60}$

Our current understanding of drug-induced adaptive immune responses is largely based on the hapten hypothesis and the p-i concept. The hapten hypothesis proposes that drugs, or more often reactive metabolites of the drugs, act as haptens and covalently bind to endogenous proteins to form immunogenic drug-protein adducts. These immunogenic adducts elicit either antibody or cytotoxic T-cell responses. The

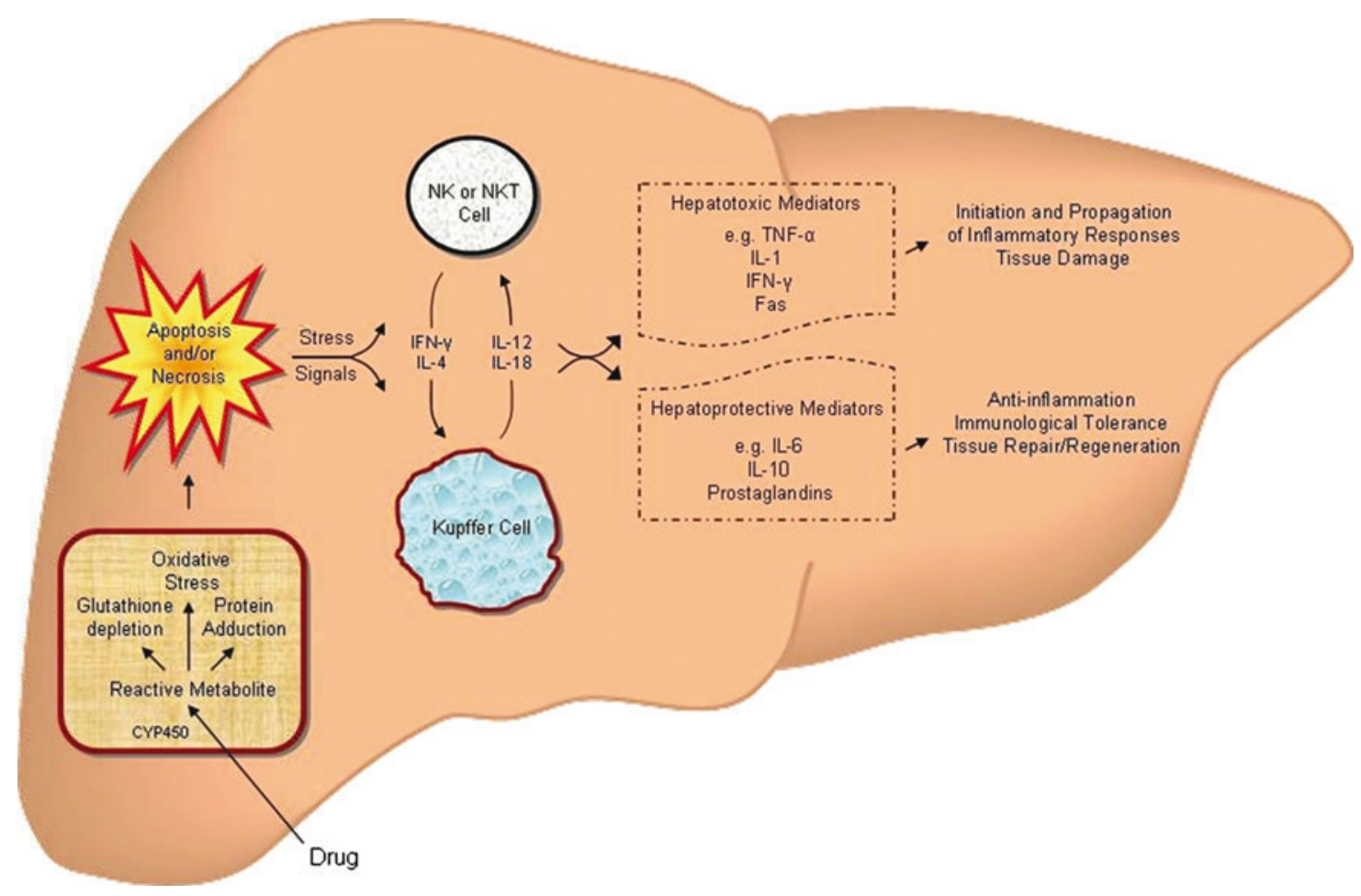

Figure 1. Illustration of the proposed mechanism of DILI, which involves drug metabolism, hepatocyte damage, activation of innate immune cells, and production of tissue-damaging and tissue-protective mediators. CYP indicates cytochrome P450; IFN, interferon; IL, interleukin; NK, natural killer cell; NKT, natural killer T cell; TNF, tumor necrosis factor. 


\section{The AAPS Journal 2006; 8 (1) Article 6 (http://www.aapsj.org).}

hapten hypothesis is supported by the detection of antibodies that recognize drug-modified hepatic proteins in the sera of DILI patients. For example, antibodies that recognize trifluoroacetate-altered hepatic proteins have been detected in the sera of patients with halothane-induced hepatitis. ${ }^{19,21}$ Such drug-specific antibodies or autoantibodies that recognize native liver proteins have also been found in patients with liver injury caused by other drugs, such as tienilic acid, dihydralazine, and diclofenac. ${ }^{15,16,61}$ The $\mathrm{p}$-i concept suggests that certain drugs can bind to T-cell receptors, mimicking a ligand and its receptor interaction, and cause T-cell activation in an MHC-dependent fashion. In patients who developed drug-induced systemic reactions of the liver and other organs, drug-specific T cells have been detected, and in some cases, T-cell clones were generated. 17,18,20

Despite the detection of drug-specific antibodies and T cells, it has been difficult to directly prove the pathogenic role of the adaptive immune system in DILI, in part because of the lack of animal models. An important reason for the difficulty in developing animal models is that the default response of the liver to antigens is immunological tolerance. This tolerogenic response could also explain the low occurrence of this type of DILI in humans. As described in the above section, the anatomy, cellular composition, and microenvironment of the liver favor tolerance rather than pathogenic immunity. Therefore, DILI mediated by adaptive immune reactions against a drug can occur only when the tolerance mechanism is deficient or abrogated in susceptible individuals. As such, animal models of this type of DILI can be developed only after the barrier of such tolerance is overcome.

\section{SumMary AND FUture PERSPECTIVES}

Because of the significant impact of DILI on public health and drug development, it is essential to understand the mechanism leading to injury in order to accurately predict and prevent this problem. Our current knowledge suggests that these reactions are initiated by hepatocyte damage and followed by a series of secondary events, including activation of innate immune cells, release of inflammatory cytokines and chemokines, and elicitation of drug-specific T- and B-cell responses. An effective means toward better understanding the molecular and cellular basis of these events is the development of animal models of DILI, in which one or more pathways described above account for the mechanism.

An important part of preventing DILI is identifying patients who are at an elevated risk of developing these reactions. The low incidence of DILI suggests that polymorphisms within a single gene cannot account for a patient's susceptibility; therefore, multiple genetic and environmental factors must converge to influence the occurrence of DILI. Widening the search for risk factors using global genomic and proteomic approaches may prove to be fruitful in the identification of this at-risk population. The current challenge we face is collecting a significant amount of patient samples. As an initial effort to solve this problem, the National Institute of Diabetes and Digestive and Kidney Diseases has created the Drug-Induced Liver Injury Network (http://dilin.dcri.duke.edu). The goals of this network include creating a registry of documented DILI cases and a bank of biological specimens, which will hopefully facilitate the identification of clinical, immunological, and environmental risk factors of DILI. It is believed that research conducted by these multicenter, multidisciplinary collaborations will eventually result in breakthrough developments in the prediction, diagnosis, and prevention of DILI.

\section{REFERENCES}

1. Ostapowicz G, Fontana RJ, Schiodt FV, et al. Results of a prospective study of acute liver failure at 17 tertiary care centers in the United States. Ann Intern Med. 2002;137:947-954.

2. Zimmerman HJ. Drug-induced liver disease. In: Zimmerman HJ, ed. Hepatotoxicity: The Adverse Effects of Drugs and Other Chemicals on the Liver. 2nd ed. Philadelphia, PA: Lippincott Williams \& Wilkins; 1999:427-456.

3. Hunter EB, Johnston PE, Tanner G, Pinson CW, Awad JA. Bromfenac (Duract)-associated hepatic failure requiring liver transplantation. Am J Gastroenterol. 1999;94:2299-2301.

4. Anonymous. Withdrawal of medicinal products containing ebrotidine: liver toxicity. WHO Inform Exchange Syst. 1998;72:1.

5. Kohlroser J, Mathai J, Reichheld J, Banner BF, Bonkovsky HL. Hepatotoxicity due to troglitazone: report of two cases and review of adverse events reported to the United States Food and Drug Administration. Am J Gastroenterol. 2000;95:272-276.

6. Lasser KE, Allen PD, Woolhandler SJ, Himmelstein DU, Wolfe SM, Bor DH. Timing of new black box warnings and withdrawals for prescription medications. JAMA. 2002;287:2215-2220.

7. Thames G. Drug-induced liver injury: what you need to know. Gastroenterol Nurs. 2004;27:31-33.

8. Temple RJ, Himmel MH. Safety of newly approved drugs: implications for prescribing. JAMA. 2002;287:2273-2275.

9. Blazka ME, Wilmer JL, Holladay SD, Wilson RE, Luster MI. Role of proinflammatory cytokines in acetaminophen hepatotoxicity. Toxicol Appl Pharmacol. 1995;133:43-52.

10. Blazka ME, Elwell MR, Holladay SD, Wilson RE, Luster MI. Histopathology of acetaminophen-induced liver changes: role of interleukin 1 alpha and tumor necrosis factor alpha. Toxicol Pathol. 1996;24:181-189.

11. Ishida Y, Kondo T, Ohshima T, Fujiwara H, Iwakura Y, Mukaida N. A pivotal involvement of IFN-gamma in the pathogenesis of acetaminophen-induced acute liver injury. FASEB J. 2002;16:1227-1236.

12. Bourdi M, Masubuchi Y, Reilly TP, et al. Protection against acetaminophen-induced liver injury and lethality by interleukin 10: role of inducible nitric oxide synthase. Hepatology. 2002;35:289-298.

13. Masubuchi Y, Bourdi M, Reilly TP, Graf ML, George JW, Pohl LR. Role of interleukin-6 in hepatic heat shock protein expression and protection against acetaminophen-induced liver disease. Biochem Biophys Res Commun. 2003;304:207-212. 


\section{The AAPS Journal 2006; 8 (1) Article 6 (http://www.aapsj.org).}

14. Reilly TP, Brady JN, Marchick MR, et al. A protective role for cyclooxygenase-2 in drug-induced liver injury in mice. Chem Res Toxicol. 2001;14:1620-1628.

15. Bourdi M, Tinel M, Beaune PH, Pessayre D. Interactions of dihydralazine with cytochromes P4501A: a possible explanation for the appearance of anti-cytochrome P4501A2 autoantibodies. Mol Pharmacol. 1994;45:1287-1295.

16. Lecoeur S, Andre C, Beaune PH. Tienilic acid-induced autoimmune hepatitis: anti-liver and -kidney microsomal type 2 autoantibodies recognize a three-site conformational epitope on cytochrome P4502C9. Mol Pharmacol. 1996;50:326-333.

17. Naisbitt DJ, Farrell J, Wong G, et al. Characterization of drugspecific T cells in lamotrigine hypersensitivity. J Allergy Clin Immunol. 2003;111:1393-1403.

18. Naisbitt DJ, Britschgi M, Wong G, et al. Hypersensitivity reactions to carbamazepine: characterization of the specificity, phenotype, and cytokine profile of drug-specific T cell clones. Mol Pharmacol. 2003;63:732-741.

19. Satoh H, Martin BM, Schulick AH, Christ DD, Kenna JG, Pohl LR. Human anti-endoplasmic reticulum antibodies in sera of patients with halothane-induced hepatitis are directed against a trifluoroacetylated carboxylesterase. Proc Natl Acad Sci USA. 1989;86:322-326.

20. Schnyder B, Mauri-Hellweg D, Zanni M, Bettens F, Pichler WJ. Direct, MHC-dependent presentation of the drug sulfamethoxazole to human alphabeta T cell clones. J Clin Invest. 1997;100:136-141.

21. Vergani D, Mieli-Vergani G, Alberti A, et al. Antibodies to the surface of halothane-altered rabbit hepatocytes in patients with severe halothane-associated hepatitis. N Engl J Med. 1980;303:66-71.

22. Larrey D. Drug-induced liver diseases. J Hepatol. 2000;32:77-88.

23. Gunawan B, Kaplowitz N. Clinical perspectives on xenobioticinduced hepatotoxicity. Drug Metab Rev. 2004;36:301-312.

24. Zimmerman HJ. Drug-induced liver disease. Clin Liver Dis. 2000;4:73-96.

25. Pessayre O, Larrey D, Biour M. Drug-induced liver injury. In: Bircher J, Benhamou J, McIntyre N, eds. Oxford Textbook of Clinical Hepatology. Oxford, England: Oxford University Press;

1999:1261-1315.

26. Lee WM. Drug-induced hepatotoxicity. $N$ Engl J Med. 2003;349:474-485.

27. Mitchell JR, Jollow DJ, Potter WZ, Davis DC, Gillette JR, Brodie BB. Acetaminophen-induced hepatic necrosis, I: role of drug metabolism. J Pharmacol Exp Ther. 1973;187:185-194.

28. Donnelly PJ, Walker RM, Racz WJ. Inhibition of mitochondrial respiration in vivo is an early event in acetaminophen-induced hepatotoxicity. Arch Toxicol. 1994;68:110-118.

29. Meyers LL, Beierschmitt WP, Khairallah EA, Cohen SD.

Acetaminophen-induced inhibition of hepatic mitochondrial respiration in mice. Toxicol Appl Pharmacol. 1988;93:378-387.

30. Li Z, Diehl AM. Innate immunity in the liver. Curr Opin Gastroenterol. 2003;19:565-571.

31. Hashimoto W, Takeda K, Anzai R, et al. Cytotoxic NK1.1 Ag+ alpha beta T cells with intermediate TCR induced in the liver of mice by IL-12. J Immunol. 1995;154:4333-4340.

32. Tsutsui H, Matsui K, Kawada N, et al. IL-18 accounts for both TNFalpha- and Fas ligand-mediated hepatotoxic pathways in endotoxininduced liver injury in mice. J Immunol. 1997;159:3961-3967.

33. Rubinstein D, Roska AK, Lipsky PE. Liver sinusoidal lining cells express class II major histocompatibility antigens but are poor stimulators of fresh allogeneic T lymphocytes. J Immunol. 1986;137:1803-1810.

34. Callery MP, Kamei T, Flye MW. Kupffer cell blockade inhibits induction of tolerance by the portal venous route. Transplantation. 1989;47:1092-1094.

35. Kamei T, Callery MP, Flye MW. Kupffer cell blockade prevents induction of portal venous tolerance in rat cardiac allograft transplantation. J Surg Res. 1990;48:393-396.

36. Squiers EC, Salomon DR, Pickard LL, Howard RR, Pfaff WW. Abrogation of the induction of portal venous tolerance in a cardiac transplant model resulting from Kupffer cell inhibition by gadolinium. Transplantation. 1990;50:171-173.

37. Mehal WZ, Azzaroli F, Crispe IN. Immunology of the healthy liver: old questions and new insights. Gastroenterology. 2001;120:250-260.

38. Doherty DG, Norris S, Madrigal-Estebas L, et al. The human liver contains multiple populations of NK cells, $\mathrm{T}$ cells, and CD3+CD56+

natural $\mathrm{T}$ cells with distinct cytotoxic activities and Th1, Th2, and Th0 cytokine secretion patterns. J Immunol. 1999;163:2314-2321.

39. Chen H, Paul WE. Cultured NK1.1+ CD4+ T cells produce large amounts of IL-4 and IFN-gamma upon activation by anti-CD3 or CD1. J Immunol. 1997;159:2240-2249.

40. Doherty DG, O'Farrelly C. Innate and adaptive lymphoid cells in the human liver. Immunol Rev. 2000;174:5-20.

41. Paul WE, Seder RA. Lymphocyte responses and cytokines. Cell. 1994;76:241-251.

42. Calne RY, Sells RA, Pena JR, et al. Induction of immunological tolerance by porcine liver allografts. Nature. 1969;223:472-476.

43. Gorczynski RM, Chan Z, Chung S, et al. Prolongation of rat small bowel or renal allograft survival by pretransplant transfusion and/or by varying the route of allograft venous drainage. Transplantation. 1994;58:816-820.

44. Rao VK, Burris DE, Gruel SM, Sollinger HW, Burlingham WJ. Evidence that donor spleen cells administered through the portal vein prolong the survival of cardiac allografts in rats. Transplantation. 1988;45:1145-1146.

45. Cantor HM, Dumont AE. Hepatic suppression of sensitization to antigen absorbed into the portal system. Nature. 1967;215:744-745.

46. Chen Y, Ong CR, McKenna GJ, Mui AL, Smith RM, Chung SW. Induction of immune hyporesponsiveness after portal vein immunization with ovalbumin. Surgery. 2001;129:66-75.

47. Crispe IN, Dao T, Klugewitz K, Mehal WZ, Metz DP. The liver as a site of T-cell apoptosis: graveyard, or killing field? Immunol Rev. 2000;174:47-62.

48. Bertolino P, Heath WR, Hardy CL, Morahan G, Miller JF. Peripheral deletion of autoreactive CD8+ T cells in transgenic mice expressing $\mathrm{H}-2 \mathrm{~Kb}$ in the liver. Eur J Immunol. 1995;25:1932-1942.

49. Huang L, Soldevila G, Leeker M, Flavell R, Crispe IN. The liver eliminates $\mathrm{T}$ cells undergoing antigen-triggered apoptosis in vivo. Immunity. 1994;1:741-749.

50. Klugewitz K, Blumenthal-Barby F, Schrage A, Knolle PA, Hamann A, Crispe IN. Immunomodulatory effects of the liver: deletion of activated CD4+ effector cells and suppression of IFN-gamma-producing cells after intravenous protein immunization. J Immunol. 2002;169:2407-2413.

51. Knolle PA, Schmitt E, Jin S, et al. Induction of cytokine production in naive $\mathrm{CD} 4(+) \mathrm{T}$ cells by antigen-presenting murine liver sinusoidal endothelial cells but failure to induce differentiation toward Th1 cells. Gastroenterology. 1999;116:1428-1440. 


\section{The AAPS Journal 2006; 8 (1) Article 6 (http://www.aapsj.org).}

52. Limmer A, Ohl J, Kurts C, et al. Efficient presentation of exogenous antigen by liver endothelial cells to CD8+ T cells results in antigenspecific T-cell tolerance. Nat Med. 2000;6:1348-1354.

53. Pillarisetty VG, Shah AB, Miller G, Bleier JI, DeMatteo RP. Liver dendritic cells are less immunogenic than spleen dendritic cells because of differences in subtype composition. J Immunol. 2004;172:1009-1017.

54. Liu ZX, Govindarajan S, Kaplowitz N. Innate immune system plays a critical role in determining the progression and severity of acetaminophen hepatotoxicity. Gastroenterology. 2004;127:1760-1774.

55. Laskin DL, Gardner CR, Price VF, Jollow DJ. Modulation of macrophage functioning abrogates the acute hepatotoxicity of acetaminophen. Hepatology. 1995;21:1045-1050.

56. Michael SL, Pumford NR, Mayeux PR, Niesman MR, Hinson JA. Pretreatment of mice with macrophage inactivators decreases acetaminophen hepatotoxicity and the formation of reactive oxygen and nitrogen species. Hepatology. 1999;30:186-195.
57. Ju C, Reilly TP, Bourdi M, et al. Protective role of Kupffer cells in acetaminophen-induced hepatic injury in mice. Chem Res Toxicol. 2002;15:1504-1513.

58. Lawson JA, Farhood A, Hopper RD, Bajt ML, Jaeschke H. The hepatic inflammatory response after acetaminophen overdose: role of neutrophils. Toxicol Sci. 2000;54:509-516.

59. Smith GS, Nadig DE, Kokoska ER, Solomon H, Tiniakos DG, Miller TA. Role of neutrophils in hepatotoxicity induced by oral acetaminophen administration in rats. J Surg Res. 1998;80:252-258.

60. Zimmerman HJ. Drug-induced liver disease. In: Schiff E, Sorrell M, Maddrey W, eds. Schiff's Diseases of the Liver. Philadelphia, PA: Lippincott-Raven; 1999:973-1064.

61. Aithal GP, Ramsay L, Daly AK, et al. Hepatic adducts, circulating antibodies, and cytokine polymorphisms in patients with diclofenac hepatotoxicity. Hepatology. 2004;39:1430-1440. 